\title{
Assisted Compatibility, and Balanced Regulation of the Mechanical, Thermal, and Antioxidant Activity of Polyvinyl Alcohol-Chinese Bayberry Tannin Extract Films Using Different Di-Aldehydes as Cross-Linkers
}

\author{
Jingjing Liao ${ }^{1,2}$, Jinxing $\mathrm{Li}^{2}$, Fuxian Yang ${ }^{2}$, Yan $\mathrm{Zhu}^{2}$, Hongyan Wang ${ }^{3}$, Guanben $\mathrm{Du}^{1,2, *}$, \\ Hisham Essawy ${ }^{4}$ and Xiaojian Zhou ${ }^{2, *}$ \\ ${ }^{1}$ Beijing Forestry University, Beijing, 100083, China \\ ${ }^{2}$ Yunnan Provincial Key Laboratory of Wood Adhesives and Glued Products, Southwest Forestry University, Kunming, 650224, \\ China \\ ${ }^{3}$ Zhejiang Academy of Forestry, Hangzhou, 310023, China \\ ${ }^{4}$ Department of Polymers and Pigments, National Research Centre, Cairo, 12622, Egypt \\ *Corresponding Authors: Xiaojian Zhou. Email: xiaojianzhou@hotmail.com; Guanben Du. Email: gongben9@hotmail.com \\ Received: 26 February 2021 Accepted: 07 April 2021
}

\begin{abstract}
Polyvinyl alcohol (PVA)-based films containing Chinese bayberry tannin (BT) were prepared by cross-linking using glyoxal, glutaraldehyde and dialdehyde starch, individually. The presence of cross-linkers was evident to promote the transparency and decrease the moisture content of PVA/BT films, while the water solubility stayed almost unchanged in the cross-linked PVA/BT films. All cross-linkers provided promotion of the water vapor permeability, mechanical property and thermal stability of PVA/BT films. The best water vapor barrier capacity was found in case of glutaraldehyde cross-linked PVA/BT film, while the highest tensile strength was encountered in case of glyoxal cross-linked PVA/BT films, compared with the uncross-linked films. The scavenging action of the films towards DPPH radical activity was influenced by the nature of each cross-linker. Namely, the PVA/AT film cross-linked by glutaraldehyde acquired the lowest radical scavenging activity in a certain time, suggesting that glutaraldehyde decreased the release rate of BT from PVA. Based on the experimental data, glutaraldehyde and dialdehyde starch could be more suitable for PVA/BT film preparation since they can promote the intermolecular interaction of PVA and BT to a reasonable extent.
\end{abstract}

\section{KEYWORDS}

Polyvinyl alcohol; Chinese bayberry tannin; cross-linker; mechanical performance; antioxidant activity

\section{Introduction}

In the past few decades, the need of using bio-degradable materials to substitute traditional nondegradable petroleum-based plastics has directed the research towards the investigation and use of various biopolymers in many potential applications, especially in the field of packaging [1]. Polyvinyl alcohol (PVA) is a hydrophilic water-soluble synthetic polymer, which is one of the most important biodegradable polymers in packaging applications due to its nontoxicity, excellent film-forming capacity and highly compatible nature [2,3]. As of the presence of hydroxyl groups in its polymer chain, PVA can 
be easily compounded with various natural compounds, tailoring the composite film according to various requirements and packaging concern. For instance, PVA was reported to incorporate with cellulose [4], chitosan [5-7], lignin [8,9], starch [10], essential oils [11], tara gum [12], and protein [13,14].

In recent years, phenolic compounds are gaining great attentions as active compounds to develop PVAbased active packaging films in consideration of their excellent antioxidant, anti-ultraviolet (UV), antifungal and antimicrobial capacity [15]. For instance, tea extract [7,16-19], tomato by-products extract [20], quercetin [21], tannin acid [22] and larch bark tannins [23,24] have been conducted to be incorporated with PVA for developing active films bearing antioxidant and antimicrobial functions. Among these phenolic compounds, tannins are water-soluble phenolic compounds with molecular weights between 500 and $3000 \mathrm{~g} / \mathrm{mol}$. Tannins can be chemically classified into hydrolysable tannins and condensed tannins [25]. The condensed tannins are widely distributed in nature and particularly enriched in barks of various trees, which are abundant wastes generated during timber production. The condensed tannins represent more than $90 \%$ of the worldwide production of commercial tannins, therefore, they are more attractive to develop active packaging materials with a reasonable price [25]. According to previous studies [26-28] and our recent work [29,30], condensed tannins have been used to develop polymeric composite materials, and enhancing the thermal stability and UV degradation resistance of polymers. In the work of Zhai et al. [24], the incorporation of PVA with $2 \%$ condensed tannin from larch bark provided good antioxidant and UV protection without causing obvious changes in the mechanical strength. However, deterioration of mechanical performance and alteration of morphology were generally found with increase of the phenolic compounds, mainly because of the imperfect miscibility. Examples of such effects, PVA compounded with more than $10 \%$ of tea polyphenol [18] or incorporated with more than $5 \%$ of gallic acid [21].

For improving the interfacial adhesion of PVA and natural compounds, the use the cross-linking agents should be capable of coping with the imperfect miscibility of two components and promotes the hydrophobicity of PVA-based composites films. Dialdehydes such as glyoxal and glutaraldehyde are common cross-linking agents for PVA blends because of their chemical reactivity and mild reaction conditions [31,32]. The formation of covalent bonds generally results in better mechanical performance, water resistance and thermal stability of PVA blends. For example, glyoxal cross-linked PVA/starch blends [33], glutaraldehyde cross-linked PVA/cellulose films [9], and glutaraldehyde cross-linked PVA/chitosan blends [34]. Except for the mentioned low molecular weight dialdehydes, dialdehyde starch is a promising polymeric dialdehydes due to its low toxicity. Its aldehyde groups could form covalent bonds with PVA, based on its chemical reactivity [35,36]. Although there are some reports regarding the cross-linking of PVA with dialdehydes [31,32,35], studies on the incorporation of tannins into PVA films with post cross-linking with dialdehydes are quite rare.

In present work, we focus on the effects of different crosslinkers on the opacity, moisture content, water solubility, water vapor permeability, mechanical, thermal stability, miscibility and antioxidant capacity of PVA/tannin films. Attempt to elucidate the chemical interactions of cross-linked PVA/BT films were performed using FT-IR and correlation with other techniques will be extended.

\section{Materials and Methods}

\subsection{Materials}

Industrial condensed tannin from Chinese bayberry (Myrica rubra Sieb. et Zucc.) with a purification at $72 \%$ was provided from Guangxi Wuming Company, China. Chinese bayberry tannin (BT), mainly consist of prodelphinidin with an average molecular weight of 2292 Dalton as reported elsewhere [37,38] was also used. Polyvinyl alcohol (PVA), glyoxal (40\%) and glutaraldehyde (50\%) were analytical reagents, bought from Sinopharm Chemical Reagent Co., Ltd., China. PVA has a hydrolysis degree of $98 \%-99 \%$ and a degree of polymerization of $1750 \pm 50$. Dialdehyde starch and 1,1-diphenyl-2-picrylhydrazyl 
(DPPH, 96\%) were purchased from Jinshan denaturated starch Co., Ltd. (Taian, China) and Shanghai macklin biochemical Co., Ltd. (Shanghai, China), respectively. All the materials were used without purification and the chemical structures of cross-linkers are presented in Fig. 1.

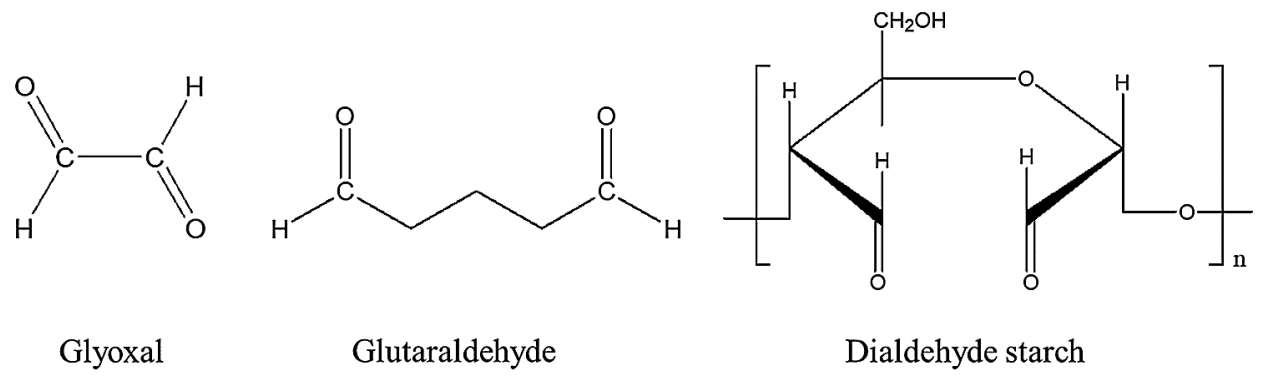

Figure 1: The main structure of the used cross-linkers

\subsection{Film Preparation}

Firstly, $5 \mathrm{wt} \%$ aqueous PVA solution was prepared at $95^{\circ} \mathrm{C}$ under magnetic stirring for more than $4 \mathrm{~h}$ to ensure it is completely dissolved. With a weight ratio of PVA and BT 9:1, the BT was dissolved in $1 \mathrm{~mL}$ distilled water before mixing with PVA. Then, 5\% glyoxal, glutaraldehyde, and dialdehyde starch as cross-linkers (based on PVA and BT) were introduced to the PVA-tannin mixtures separately. For achieving complete dissolution and dispersion, all chemicals were constantly stirred for $10 \mathrm{~min}$ at room temperature. Subsequently, the mixtures were magnetically stirred at $80^{\circ} \mathrm{C}$ for $30 \mathrm{~min}$. Finally, solutions $(80 \mathrm{~mL})$ were casted on the self-made glass plate $(21 \mathrm{~cm} \times 21 \mathrm{~cm})$ and air-dried at room temperature $\left(15-20^{\circ} \mathrm{C}\right)$ under a relative moisture of $45 \%-50 \%$ for $72 \mathrm{~h}$ for film formation. All films were peeled off and stored in desiccators for at least $24 \mathrm{~h}$ before characterizations to be undertaken. The PVA-BT film without cross-linkers was named as PT, while PVA/BT films cross-linked with glyoxal, glutaraldehyde, and dialdehyde starch were noted as PT-GO, PT-GA and PT-DS, respectively. In order to have statistical analysis, all experiments were conducted with at least three samples $(n=3)$, where the values were averaged and stander deviation (SD) was used as error on tables and bar graphs.

\subsection{Characterizations}

\subsubsection{Thickness}

A micrometer with a resolution of $0.001 \mathrm{~mm}$ (Everte, Shenzhen, China) was used to measure the thickness of obtained films. Each specimen was measured at 10 random points and average thickness was calculated.

\subsubsection{Opacity}

Based on the method of Wen et al. [17], the opacity of the films was determined by measuring the absorbance at a wavelength of $600 \mathrm{~nm}$ using a UV spectrometer (WFZUV-8802S, China). The specimens $(4 \mathrm{~cm} \times 1 \mathrm{~cm})$ were placed in a spectrophotometer cell. The opacity value was calculated according to Eq. (1):

Opacity $=\frac{\mathrm{Abs}_{600}}{\mathrm{x}}$

where, $\mathrm{Abs}_{600}$ is the absorption at $600 \mathrm{~nm}$, and $\mathrm{x}$ is the thickness. The samples were measured repeatedly for three times. 


\subsubsection{Moisture Content}

The specimens $(2 \mathrm{~cm} \times 2 \mathrm{~cm})$ were pre-weighted $\left(\mathrm{M}_{0}\right)$ and dried in an oven at $105^{\circ} \mathrm{C}$ to a constant weight $\left(\mathrm{M}_{1}\right)$. Three repetitions were performed for each assay. The moisture content $(\mathrm{MC})$ of the films was calculated using Eq. (2):

$\operatorname{MC}(\%)=\frac{\mathrm{M}_{0}-\mathrm{M}_{1}}{\mathrm{M}_{0}} \times 100$

\subsubsection{Water Solubility}

The tested samples $(2 \mathrm{~cm} \times 2 \mathrm{~cm})$ were pre-weighed $\left(\mathrm{M}_{0}\right)$ and placed in distilled water at room temperature. After $24 \mathrm{~h}$, the samples were removed and dried at $105^{\circ} \mathrm{C}\left(\mathrm{M}_{1}\right)$ for $2 \mathrm{~h}$. Three replicates were performed for each assay. The water solubility (WS) was calculated based on Eq. (3):

$\mathrm{WS}(\%)=\frac{\mathrm{M}_{0}-\mathrm{M}_{1}}{\mathrm{M}_{0}} \times 100$

\subsubsection{Water Vapor Permeability}

Water vapor permeability (WVP) was determined gravimetrically based on the approach described by Peng et al. [7] and Wen et al. [17] with some modifications. A film (80 $\mathrm{mm}$ diameter) was sealed into a permeation cell (a baker, inner diameter: $50 \mathrm{~mm}$, height: $70 \mathrm{~mm}$ ) containing $3 \mathrm{~g}$ granular anhydrous calcium chloride. Then, the covered permeation cells were placed in a desiccator containing $1 \mathrm{~L}$ distilled water, providing RH gradients of $100 \%$. The covered permeation cells were weighed every $12 \mathrm{~h}$ for 3 consecutive days at room temperature. The WVP was calculated using Eq. (4):

$\mathrm{WVP}=\frac{\mathrm{W} \times \mathrm{x}}{\mathrm{A} \times \Delta \mathrm{P} \times \mathrm{t}}$

where $\mathrm{W}$ is the weight increment of the covered beaker $(\mathrm{g}), \mathrm{x}$ is the thickness of film $(\mathrm{mm}), \mathrm{t}$ is the duration (s) of the covered beaker with increased weight, $\mathrm{A}$ is the permeation area of the film sample $\left(\mathrm{m}^{2}\right)$, and $\Delta \mathrm{P}$ is the partial vapor pressure. Three repetitions were performed for each film sample.

\subsubsection{Tensile Strength}

The mechanical property of films was determined by tensile test according to GB/T 1040.3-2006. Films were cut into strips $(160 \mathrm{~mm} \times 20 \mathrm{~mm}$ ) and tested using SUST universal testing machine (model 5569, China). The initial grip separation was $50 \mathrm{~mm}$ and the cross-head speed was adjusted at $50 \mathrm{~mm} / \mathrm{min}$. Five repetitions were performed for each sample.

\subsubsection{Thermogravimetric Analysis}

The thermal degradation of films (5-10 mg) was conducted using a thermogravimetric analyzer (TGA) (TG209F1, NETZSCH, Germany). The thermal runs were performed from 30 to $600^{\circ} \mathrm{C}$ with a constant heating rate of $10^{\circ} \mathrm{C} / \mathrm{min}$ under nitrogen protection.

\subsubsection{Morphological Aspects}

Morphology of films cross-sections was observed by scanning electron microscopy (SEM) (Zeiss Sigma 300 , Germany), with an accelerating voltage of $20 \mathrm{kV}$. The films were previously frozen in liquid nitrogen, fractured, and coated with gold before observation. 


\subsubsection{Infrared Spectroscopy}

A thermo scientific Nicolet IS50 FT-IR spectrometer (China) equipped with attenuated total reflection mode was used to evaluate the chemical structure of the resulting films. The spectra of films were recorded from 4000 to $600 \mathrm{~cm}^{-1}$ at $4 \mathrm{~cm}^{-1}$ resolution with 32 scans.

\subsubsection{Antioxidant Activity}

The antioxidant activity of the films was carried out based on a previously reported method [16]. Each film $(0.3 \mathrm{~g})$ was initially soaked into $90 \mathrm{~mL}$ distilled water and magnetically stirred for $30 \mathrm{in}$. Then, $1 \mathrm{~mL}$ of the obtained solution was withdrawn to mix with $4 \mathrm{~mL}$ of DPPH- methanol solution $(150 \mathrm{~mol} / \mathrm{L})$ in dark. After $30 \mathrm{~min}$, the absorbance of the mixture was measured at $517 \mathrm{~nm}$ using an UV spectrophotometer (WFZUV-8802S, China). The DPPH radical scavenging activity was determined according to Eq. (5):

DPPH radical scavenging activity $(\%)=\left[1-\frac{\mathrm{A}_{\text {sample }}}{\mathrm{A}_{\text {control }}}\right] \times 100$

where $\mathrm{A}_{\text {control }}$ is the absorbance of the control using $1 \mathrm{~mL}$ distilled water in replace of the sample solution while $\mathrm{A}_{\text {sample }}$ is the absorbance of the tested sample. Each assay was performed in triplicate.

\section{Results and Discussion}

\subsection{Thickness, Opacity and Water Solubility}

The thickness and opacity of resulting films are shown in Tab. 1. The thickness of PVA/BT films slightly increased because the addition of BT slightly increased the contents of the film-forming solution. The opacity value corresponds to a higher visible light absorbance of a sample at a given thickness and associates with the appearance of films [17]. The opacity of PVA/BT films are generally higher than that of PVA because the addition of BT decreased the transparency of films and increased the light absorbance in the visible region. Moreover, cross-linked PVA/BT films, especially PT-GO and PT-GA, exhibit lower opacity values than PT, suggesting a better miscibility with the consideration of their microstructures from one side (as will be seen later in Section 3.5) and hindrance of the light absorbance after the chains became more tightly packed.

Table 1: Thickness, opacity, moisture content and water solubility values of PVA, PT and cross-linked PVA/ BT films

\begin{tabular}{lllll}
\hline Samples & Thickness $(\mu \mathrm{m})$ & Opacity $(\mathrm{Abs} / \mathrm{mm})$ & MC $(\%)$ & WS $(\%)$ \\
\hline PVA & $67.25 \pm 11.63$ & $1.53 \pm 0.17$ & $9.98 \pm 0.02$ & $5.58 \pm 0$ \\
PT & $68.00 \pm 8.96$ & $2.56 \pm 0.24$ & $11.27 \pm 0.01$ & $9.58 \pm 0.05$ \\
PT-GO & $81.59 \pm 6.89$ & $2.05 \pm 0.30$ & $9.59 \pm 0.16$ & $13.83 \pm 0.02$ \\
PT-GA & $70.25 \pm 5.89$ & $2.22 \pm 0.22$ & $10.56 \pm 0.86$ & $14.57 \pm 1.50$ \\
PT-DS & $78.38 \pm 8.83$ & $2.51 \pm 0.13$ & $10.10 \pm 0.22$ & $15.18 \pm 0.60$ \\
\hline
\end{tabular}

Note: Values are given as mean $\pm \mathrm{SD}(\mathrm{n}=3)$.

The corresponding data of moisture content (MC) and water solubility (WS) are listed in Tab. 1. The moisture content of materials is related to the hydroxyl groups of polymer chains and microstructure of the obtained films [39]. The higher MC value of PT with respect to PVA is caused probably by an extent of mismatching between the components, resulting in free volume, as can be observed in the fractured cross-section of PT (as will be seen later in Section 3.5). With the addition of cross-linkers, the MC of PVA/BT films decreased slightly due to the cross-linking reactions and the improved interfacial adhesion 
of PVA and BT. The WS of PVA/BT films were generally higher than that of PVA considering free tannin release when films are immersed in water, which was also reported in the study of Dai et al. [22]. It should be noted that the cross-linked PVA/BT films display higher WS values compared with PT, suggesting that more free tannin is presented in the cross-linked PVA/BT films. It is well known that hydroxyl groups from PVA react with aldehydes via formation of acetal bonds, these cross-linking reactions on PVA can be conducted under mild conditions [32]. However, tannin reacts with aldehydes via electrophilic aromatic substitution, which is less reactive under mild conditions [40]. Thus, the cross-linking reactions take place mainly within PVA chains rather than at the interface between PVA and tannin.

\subsection{Water Vapor Permeability}

Water vapor permeability (WVP) refers to the capacity of water vapors that can diffuse through a film [41], which depends on the intermolecular interactions and morphology of films [39]. The WVP of PVA, PT and cross-linked PVA/BT films are given in Fig. 2. PVA acquired a value of $1.59 \times 10^{-7} \mathrm{gs}^{-1} \mathrm{~m}^{-1} \mathrm{~Pa}^{-1}$, while in case of PT it decreased to $1.27 \times 10^{-7} \mathrm{gs}^{-1} \mathrm{~m}^{-1} \mathrm{~Pa}^{-1}$, indicating that the hydrogen bonds between PVA and BT postponed the diffusion of water vapors through the film. All cross-linked PVA/BT films exhibited lower WVP values compared with PVA since a major part of the hydroxyl groups of PVA have been consumed in reaction with the aldehyde groups of the cross-linkers, resulting in less hydrophilic material [31,32]. Besides, the presence of cross-linkers promoted the compatibility between PVA and BT, hence the good intermolecular interactions between tannin and PVA formed more tortuous paths [8]. Additionally, the bulky nature of tannin might serve as physical barriers in the PVA polymer chain, a trend that is commonly found in nanoparticles filled PVA system [8]. The vibrations of WVP for PVA/BT films crosslinked with different dialdehydes might have been caused by the difference in chemical reactivity and molecular size of each dialdehyde. Glyoxal, the smallest dialdehyde, could be easier to cross-link PVA chains since it can easily fit into the void space [41]. A relatively intense cross-linked structure could be formed in the glyoxal cross-linked PVA/BT film, which is generally not favorable for water vapor permeation through the film unless considering pores formation.

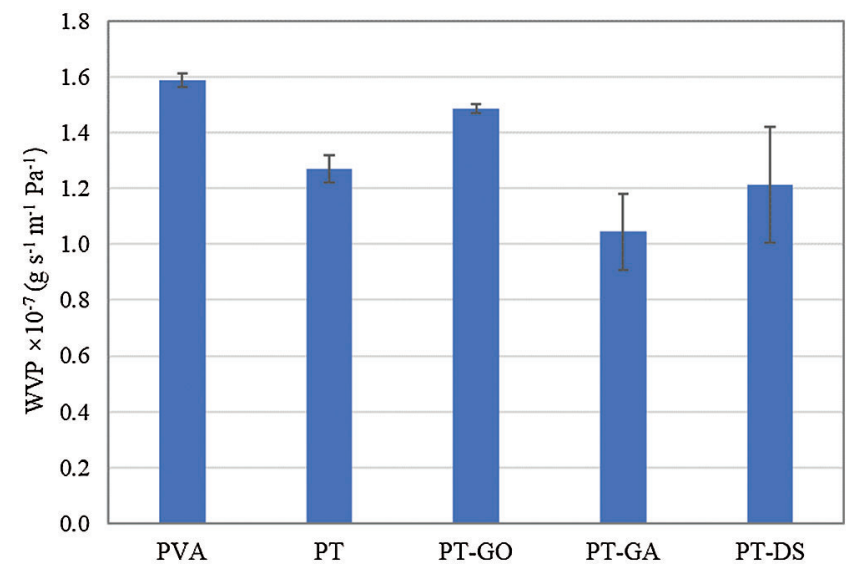

Figure 2: The water vapor permeability within PVA, PT and cross-linked PVA/BT films. Each values represents mean $\pm \mathrm{SD}(\mathrm{n}=3)$

\subsection{Tensile Strength}

Fig. 3 presents the tensile strength and elongation at break of PVA and PVA/BT films, which are the most important properties for packaging materials. The PVA had a tensile strength of $30.1 \mathrm{MPa}$ and elongation of 412.5\%. The elongation of PT is comparable to that of PVA, however, the tensile strength of PT declined to 
22.1 MPa. This can be accounted for by a possible reduction of crystalline regions in PVA by the addition of the bulky tannin, which is commonly found in polymer blend systems [22]. In addition, the intermolecular interaction of PT film mainly depends on the intermolecular hydrogen bonding interaction with an interpenetrated network, an outer force easily results in sliding of polymer chains. Similar results can be found in PVA/gallic acid film [21] and PVA/tea polyphenol film [18]. The tensile strength values of PVA/BT films cross-linked by glyoxal, glutaraldehyde and dialdehyde starch significantly increased from 22.1 MPa to 42.2 $\mathrm{MPa}, 37.4 \mathrm{MPa}$ and $29.4 \mathrm{MPa}$, respectively, compared with that of uncross-linked PVA/BT film. This suggests that cross-linking reactions took place via stronger covalent bonding on the expense of hydrogen bonding, rendering the sliding of polymer chains more difficult in the cross-linked films. This translates into higher rigidity and consequently the elongation at break of cross-linked PVA/BT films decreased. It is also clear that the inherent rigidity of BT played a role in this context. As presented in Fig. 3, the tensile strength of PT-GO is higher than PT-GA and PT-DS, indicating that glyoxal generated relatively more efficient cross-linked structure in PVA/BT blending system due to its smaller size and higher reactivity with respect to other investigated aldehydes.

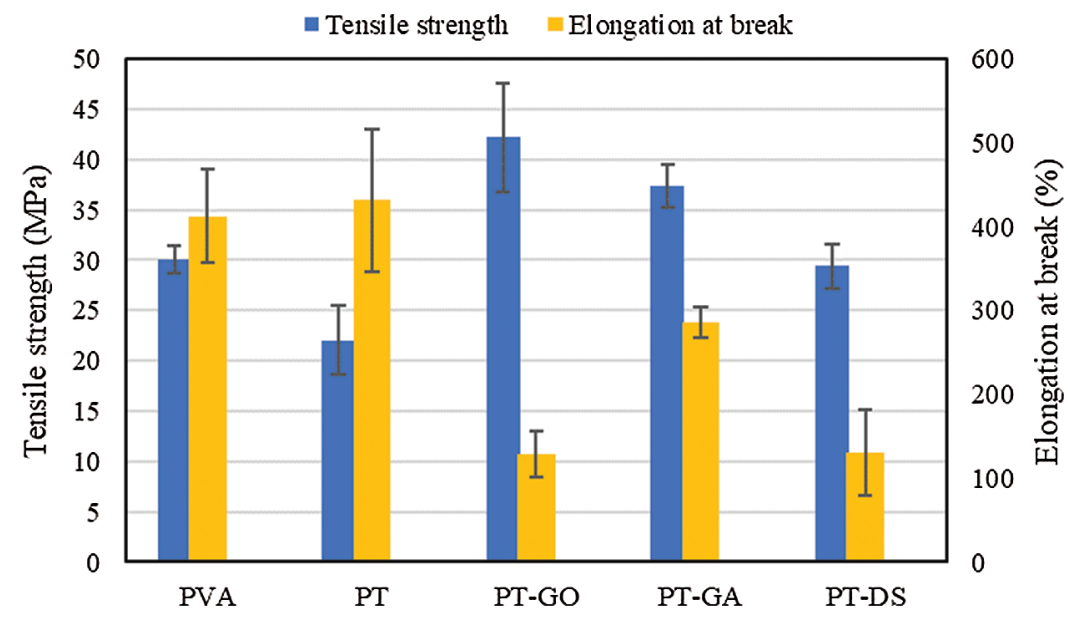

Figure 3: Tensile strength and elongation at rupture for PVA and PVA/BT films before and after crosslinking. Each value represents mean $\pm \mathrm{SD}(\mathrm{n}=5)$

\subsection{Thermal Stability}

The TG and DTG curves of PVA, PT and cross-linked PVA/BT films are presented in Fig. 4, showing the effect of cross-linkers on the resistance of PVA/BT films to heat-induced degradation. Three consecutive degradation steps were observed in all samples as previously observed [8,21]. The first stage ranges from 50 to $150^{\circ} \mathrm{C}$ with approximately $10 \%$ weight loss, related to the release of adsorbed/bound water and volatile compounds from the films. The maximum weight loss was undergone in the second stage at $200-350^{\circ} \mathrm{C}$, corresponding to the breakage of the polymer chains. The third step (350 to $600^{\circ} \mathrm{C}$ ) comprised the cleavage of the $\mathrm{C}-\mathrm{C}$ backbone of the PVA and production of carbon molecules and hydrocarbons. As can be found from DTG curves of Fig. 4, the peak 1 of PVA/BT films shifted to higher temperature with the addition of BT and cross-linkers. This suggests that the dispersion of BT in PVA and cross-linked structure presented additional protection against the effect of heat on the films, delaying the removal of water and residual acetic acid. Similar trend can be found for peak 2 in case of PVA/BT films and their weight residues are higher than that of PVA, implying carbon residues due to presence of BT. Approximately $10 \%$ residual weight can be found in PVA/BT films since the char forming capacity of tannin. This result is encountered generally in the polymeric composites containing phenolic 
compounds $[8,42]$. Besides, the residual mass for PT-GA or the initial decomposition temperature of PT-GO and PT-DS in the second stage were further upgraded with respect to PT, indicating an enhanced thermal stability because of the stable cross-linked network and the promoted intermolecular interactions of PVA and BT.

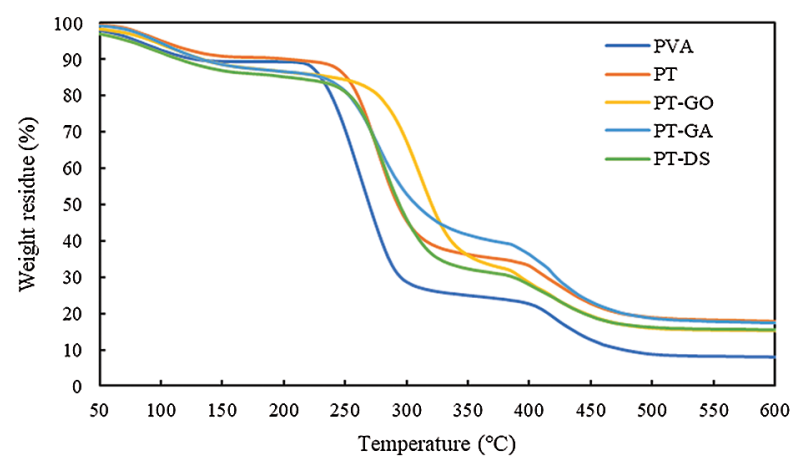

(a)

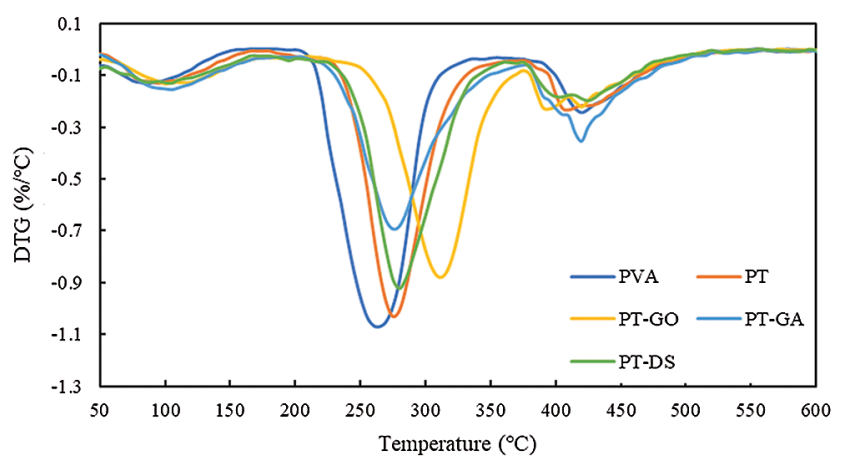

(b)

Figure 4: TG (a) and DTG (b) curves of PVA, PT and cross-linked PVA/BT films

\subsection{Morphology}

Fig. 5 displays the SEM images of cross-sections from PVA, PT and cross-linked PVA/BT films at two different magnifications. PVA shows a smooth and uniform fractured surface. However, with the addition of BT, the fracture surface of PT became rough due to limited phase separation, which can be observed obviously in some regions of the fractured surface (marked with white arrows), indicating the weak intermolecular interactions between PVA and BT. The cross-linked PVA/BT films show a continuous, homogeneous phase with no visible phase separation. This implies that either glyoxal, or glutaraldehyde, or dialdehyde starch could promote the miscibility of PVA and BT.

\subsection{FTIR Analysis}

The FTIR spectra of PVA, PT and cross-linked PVA/BT films are shown in Fig. 6. A broad band near $3400 \mathrm{~cm}^{-1}$ is ascribed to -OH stretching mainly from PVA. The peaks at $2970 \mathrm{~cm}^{-1}$ and $2893 \mathrm{~cm}^{-1}$ are typical stretching of C-H from PVA backbone. The peak at $1659 \mathrm{~cm}^{-1}$ is assigned to $\mathrm{C}=\mathrm{O}$ stretching from PVA units. The two peaks at $1430 \mathrm{~cm}^{-1}$ and $1312 \mathrm{~cm}^{-1}$ are associated with $-\mathrm{CH}_{2}$ bending. The band at $1066 \mathrm{~cm}^{-1}$ represents the $\mathrm{C}-\mathrm{O}$ stretching vibration of PVA. A shift can be found for the -OH band of PT-GA and PT-DS, probably caused by the induced change of intermolecular hydrogen bonds and limited crosslinking reactions [36]. The reduced intensity of $-\mathrm{OH}$ band found in case of PT and PT-GO, suggests elimination of majority of hydroxyl groups. This is already evidenced due to intermolecular hydrogen bonds of PVA-tannin and PVA cross-linked with glyoxal [32]. Neither new peaks are generated nor any peak was depleted in the resulting spectra, implying that the chemical reaction between PVA and tannin was limited at the employed mild reacting conditions [31,32]. This is matching with the involvement of tannin as a minor additive into a macromolecular matrix such as PVA and the major involvement of interactions of the hydroxyl groups from both sides, either in presence or absence of the crosslinkers. 

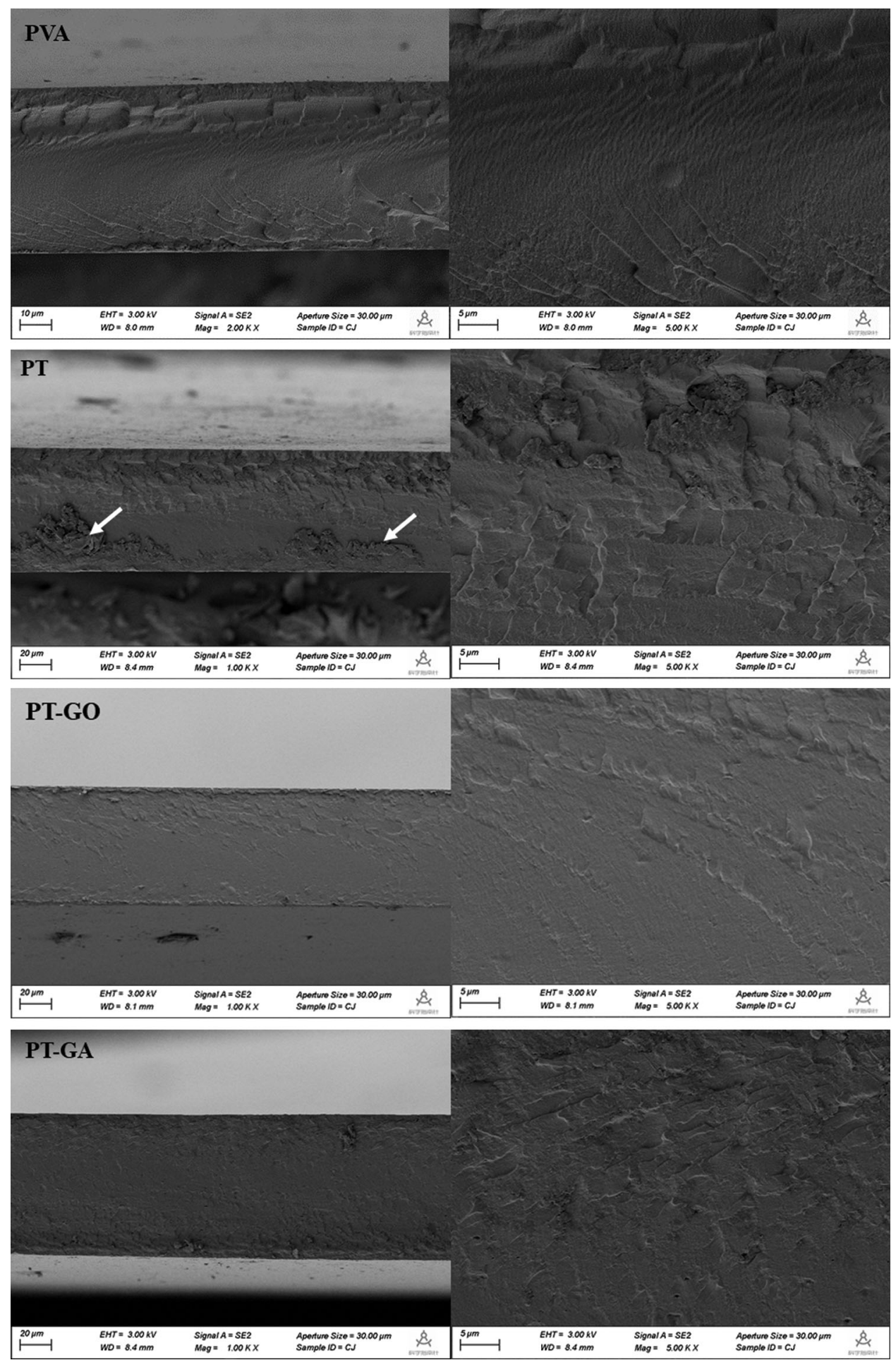

Figure 5: (continued) 


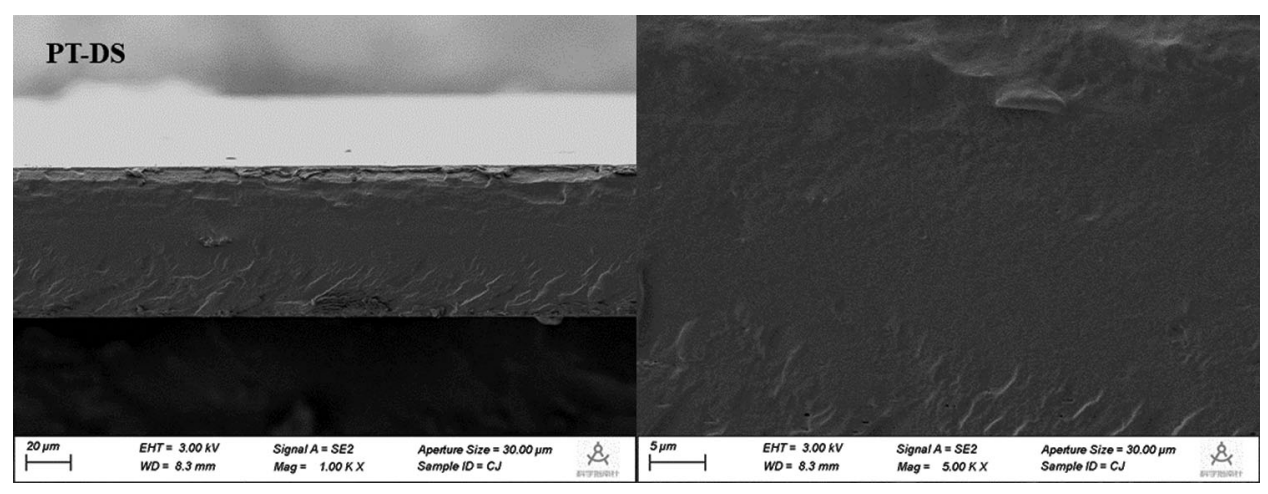

Figure 5: Fractured surface cross-sections of PVA, PT and cross-linked PVA/BT films

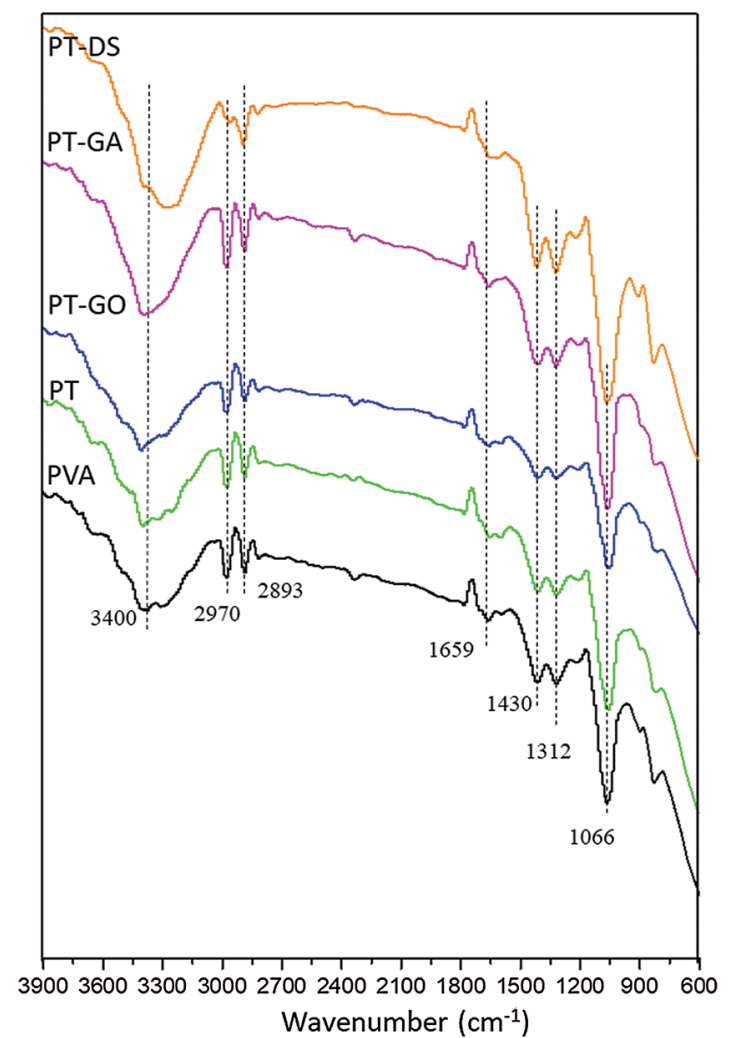

Figure 6: FTIR spectra of PVA, PT and cross-linked PVA/BT films

\subsection{Antioxidant Capacity}

The antioxidant activity of the films was measured through DPPH assay, which is one of the most widely used methods for antioxidant capacity evaluation [43]. Fig. 7 illustrates the DPPH radical scavenging activity of the resulting films. PVA alone presented slightly DPPH radical scavenging activity $(\sim 10 \%)$ owing to the presence of hydrogen on the carbon atom with hydroxyl groups as reported by Luo [43]. The DPPH scavenging activity of PT increased markedly from $10 \%$ to $67 \%$, associating with the release of tannin from PVA matrix. This behavior is related to the inner structure of film and the intermolecular interaction of active compounds with polymer matrix. As can be found in Fig. 7, PVA/BT film cross-linked by glyoxal exhibits the highest radical scavenging activity, following by PT-DS, PT and PT-GA, suggesting 
that tannin can be easier released from PT-GO film. This confirms that the cross-linking reactions initiated by glyoxal mainly occurred in PVA polymer chains rather than PVA-tannin. Thus, relatively less interaction between PVA and tannin results in fast release of tannin. By contrast, the lowest radical scavenging activity can be found in glutaraldehyde cross-linked PVA/AT film, implying that glutaraldehyde can promote the chemical reaction of PVA and tannin. The enhanced intermolecular interaction of PVA and BT slows down the release rate of BT from PVA, which will contribute to its application as active packaging materials.

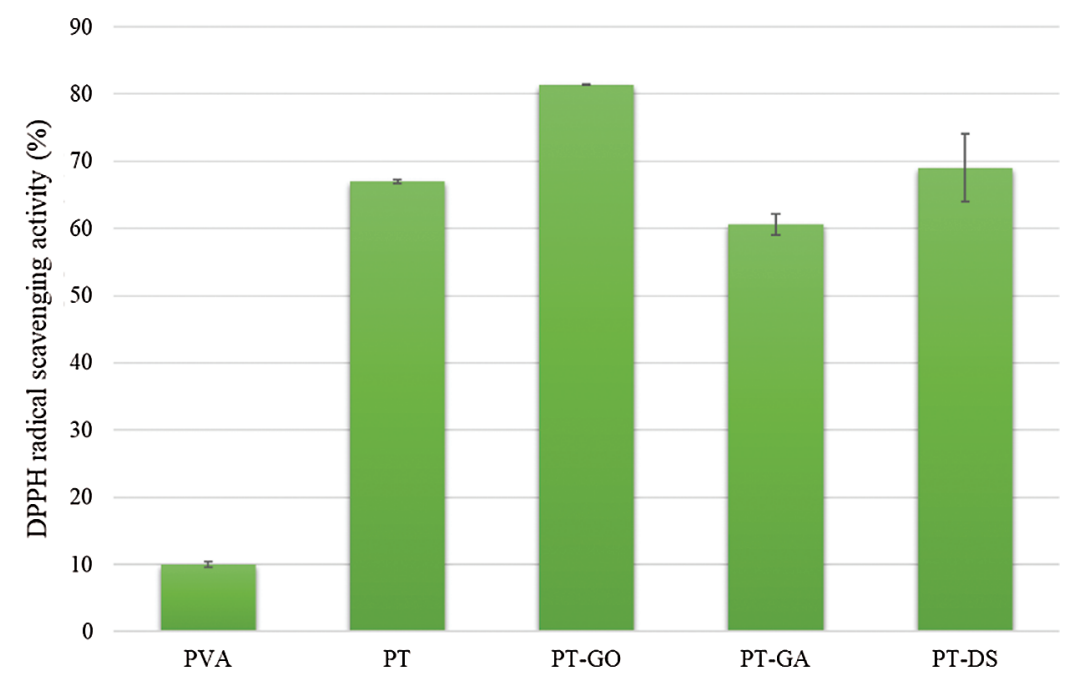

Figure 7: DPPH radical scavenging activity of PVA, PT and cross-linked PVA/BT films. Each value represents mean $\pm \mathrm{SD}(\mathrm{n}=3)$

\section{Conclusion}

PVA-based films containing Chinese bayberry tannin (BT) can be cross-linked using either glyoxal, glutaraldehyde or dialdehyde starch. Transparency, reasonable water vapor barrier and low moisture content can be ensured for the films in presence of the cross-linkers. The impact of the cross-linkers can be extended to a significant improvement of mechanical strength, especially in case of glyoxal due to its small molecular size and consequently the thermal stability was also upgraded, implying that the intermolecular interactions between PVA and tannin are enhanced in presence of such cross-linkers. This can be indirectly supported by appearance of the fracture surface of these films continuous and homogeneous using microscopic investigation. The cross-linkers played a major role in controlling the radical scavenging activity of the films. Definitely, a higher extent of intermolecular interactions between PVA and BT in presence of a cross-linker responses longer delay of the release rate of BT and thereby causes lower scavenging action towards radicals in a definite time. In all, glutaraldehyde and dialdehyde starch can be considered best suitable cross-linkers for PVA/BT films in the sense of offering reasonable well-balanced overall properties.

Funding Statement: This work was supported by the National Natural Science Foundation of China (NSFC 31971595, 31760187), the Program for Leading Talents of Science and Technology (Grant No. 2017HA013), the Yunnan Provincial Reserve Talents for Middle \& Young Academic and Technical Leaders (2019HB026), and the 111 Project.

Conflicts of Interest: The authors declare that they have no conflicts of interest to report regarding the present study. 


\section{References}

1. Rodríguez-Rojas, A., Arango Ospina, A., Rodríguez-Vélez, P., Arana-Florez, R. (2019). What is the new about food packaging material? A bibliometric review during 1996-2016. Trends in Food Science \& Technology, 85, 252-261. DOI 10.1016/j.tifs.2019.01.016.

2. Aslam, M., Kalyar, M. A., Raza, Z. A. (2018). Polyvinyl alcohol: A review of research status and use of polyvinyl alcohol based nanocomposites. Polymer Engineering \& Science, 58, 2119-2132. DOI 10.1002/pen.24855.

3. Halima, N. B. (2016). Poly(vinyl alcohol): Review of its promising applications and insights into biodegradation. RSC Advances, 6, 39823-39832. DOI 10.1039/C6RA05742J.

4. Cazón, P., Vázquez, M., Velazquez, G. (2018). Cellulose-glycerol-polyvinyl alcohol composite films for food packaging: Evaluation of water adsorption, mechanical properties, light-barrier properties and transparency. Carbohydrate Polymers, 195, 432-443. DOI 10.1016/j.carbpol.2018.04.120.

5. Balasubramaniam, M. P., Murugan, P., Chenthamara, D., Ramakrishnan, S. G., Salim, A. et al. (2020). Synthesis of chitosan-ferulic acid conjugated poly(vinyl alcohol) polymer film for an improved wound healing. Materials Today Communications, 25, 101510. DOI 10.1016/j.mtcomm.2020.101510.

6. Haghighi, H., Leugoue, S. K., Pfeifer, F., Siesler, H. W., Licciardello, F. et al. (2020). Development of antimicrobial films based on chitosan-polyvinyl alcohol blend enriched with ethyl lauroylarginate (LAE) for food packaging applications. Food Hydrocolloids, 100, 105419. DOI 10.1016/j.foodhyd.2019.105419.

7. Peng, Y., Wu, Y., Li, Y. (2013). Development of tea extracts and chitosan composite films for active packaging materials. International Journal of Biological Macromolecules, 59, 282-289. DOI 10.1016/j. ijbiomac.2013.04.019.

8. Yang, W., Owczarek, J. S., Fortunati, E., Kozanecki, M., Mazzaglia, A. et al. (2016). Antioxidant and antibacterial lignin nanoparticles in polyvinyl alcohol/chitosan films for active packaging. Industrial Crops and Products, 94, 800-811. DOI 10.1016/j.indcrop.2016.09.061.

9. Yang, W., Qi, G., Kenny, J. M., Puglia, D., Ma, P. (2020). Effect of cellulose nanocrystals and lignin nanoparticles on mechanical, antioxidant and water vapour barrier properties of glutaraldehyde crosslinked PVA films. Polymers, 12, 1364. DOI 10.3390/polym12061364.

10. Das, K., Ray, D., Bandyopadhyay, N. R., Gupta, A., Sengupta, S. et al. (2010). Preparation and characterization of cross-linked starch/Poly(vinyl alcohol) green films with low moisture absorption. Industrial \& Engineering Chemistry Research, 49, 2176-2185. DOI 10.1021/ie901092n.

11. Debiagi, F., Kobayashi, R. K. T., Nakazato, G., Panagio, L. A., Mali, S. (2014). Biodegradable active packaging based on cassava bagasse, polyvinyl alcohol and essential oils. Industrial Crops and Products, 52, 664-670.

12. Ma, Q., Ren, Y., Wang, L. (2017). Investigation of antioxidant activity and release kinetics of curcumin from tara gum/polyvinyl alcohol active film. Food Hydrocolloids, 70, 286-292.

13. El-Nemr, K. F., Mohamed, H. R., Ali, M. A., Fathy, R. M., Dhmees, A. S. (2020). Polyvinyl alcohol/gelatin irradiated blends filled by lignin as green filler for antimicrobial packaging materials. International Journal of Environmental Analytical Chemistry, 100, 1578-1602.

14. DeButts, B. L., Spivey, C. R., Barone, J. R. (2018). Wheat gluten aggregates as a reinforcement for poly(vinyl alcohol) films. ACS Sustainable Chemistry \& Engineering, 6, 2422-2430.

15. Quideau, S., Deffieux, D., Douat-Casassus, C., Pouysegu, L. (2011). Plant polyphenols: Chemical properties, biological activities, and synthesis. Angewandte Chemie International Edition, 50, 586-621.

16. Chen, C. W., Tang, Z. P., Ma, Y. R., Qiu, W. Q., Yang, F. X. et al. (2018). Physicochemical, microstructural, antioxidant and antimicrobial properties of active packaging films based on poly(vinyl alcohol)/clay nanocomposite incorporated with tea polyphenols. Progress in Organic Coatings, 123, 176-184.

17. Wen, H., Hsu, Y. L., Asoh, T. A., Uyama, H. (2020). Antioxidant activity and physical properties of pH-sensitive biocomposite using poly(vinyl alcohol) incorporated with green tea extract. Polymer Degradation and Stability, $178,109215$.

18. Zhang, L., Shen, Q. (2020). Fully green poly(vinyl alcohol)/Tea polyphenol composites and super anti-ultraviolet and -bacterial properties. Macromolecular Material and Engineering, 305, 1900669. 
19. Lan, W., Zhang, R., Ahmed, S., Qin, W., Liu, Y. (2019). Effects of various antimicrobial polyvinyl alcohol/tea polyphenol composite films on the shelf life of packaged strawberries. LWT, 113, 108297.

20. Szabo, K., Teleky, B. E., Mitrea, L., Călinoiu, L. F., Martău, G. A. et al. (2020). Active packaging-Poly(vinyl alcohol) films enriched with tomato by-products extract. Coatings, 10, 141.

21. Luzi, F., Pannucci, E., Santi, L., Kenny, J. M., Torre, L. et al. (2019). Gallic acid and quercetin as intelligent and active ingredients in poly(vinyl alcohol) films for food packaging. Polymers, 11, 1999. DOI 10.3390/ polym11121999.

22. Dai, H., Huang, Y., Huang, H. (2018). Enhanced performances of polyvinyl alcohol films by introducing tannic acid and pineapple peel-derived cellulose nanocrystals. Cellulose, 25, 4623-4637. DOI 10.1007/s10570-018-1873-5.

23. da Cruz, J. A., da Silva, A. B., Ramin, B. B. S., Souza, P. R., Popat, K. C. et al. (2020). Poly(vinyl alcohol)/ cationic tannin blend films with antioxidant and antimicrobial activities. Materials Science and Engineering: C, 107, 110357. DOI 10.1016/j.msec.2019.110357.

24. Zhai, Y., Wang, J., Wang, H., Song, T., Hu, W. et al. (2018). Preparation and characterization of antioxidative and UV-protective larch bark tannin/PVA composite membranes. Molecules, 23, 2073. DOI 10.3390/ molecules23082073.

25. Arbenz, A., Avérous, L. (2015). Chemical modification of tannins to elaborate aromatic biobased macromolecular architectures. Green Chemistry, 17, 2626-2646. DOI 10.1039/C5GC00282F.

26. Bridson, J. H., Kaur, J., Zhang, Z., Donaldson, L., Fernyhough, A. (2015). Polymeric flavonoids processed with co-polymers as UV and thermal stabilisers for polyethylene films. Polymer Degradation and Stability, 122, 18-24. DOI 10.1016/j.polymdegradstab.2015.10.002.

27. Shnawa, H. A. (2017). Thermal stabilization of polyvinyl chloride with traditional and naturally derived antioxidant and thermal stabilizer synthesized from tannins. Journal of Thermal Analysis Calorimetry, 129, 789-799. DOI 10.1007/s10973-017-6238-z.

28. Grigsby, W. J., Bridson, J. H., Schrade, C. (2015). Modifying biodegradable plastics with additives based on condensed tannin esters. Journal of Applied Polymer Science, 132, 41626.

29. Liao, J., Brosse, N., Pizzi, A., Hoppe, S. (2019). Dynamically cross-linked tannin as a reinforcement of polypropylene and UV protection properties. Polymers, 11, 102. DOI 10.3390/polym11010102.

30. Liao, J., Brosse, N., Pizzi, A., Hoppe, S., Xi, X. et al. (2019). Polypropylene blend with polyphenols through dynamic vulcanization: Mechanical, rheological, crystalline, thermal, and UV protective property. Polymers, 11, 1108. DOI 10.3390/polym11071108.

31. Figueiredo, K. C. S., Alves, T. L. M., Borges, C. P. (2009). Poly(vinyl alcohol) films crosslinked by glutaraldehyde under mild conditions. Journal of Applied Polymer Science, 111, 3074-3080. DOI 10.1002/app.29263.

32. Zhang, Y., Zhu, P. C., Edgren, D. (2010). Crosslinking reaction of poly(vinyl alcohol) with glyoxal. Journal of Polymer Research, 17, 725-730. DOI 10.1007/s10965-009-9362-z.

33. Gadhave, R. V., Mahanwar, P. A., Gadekar, P. T. (2019). Study on various compositions of polyvinyl alcohol and starch blends by cross-linking with glyoxal. Open Journal of Polymer Chemistry, 9, 76. DOI 10.4236/ ojpchem.2019.94007.

34. Costa-Júnior, E. S., Barbosa-Stancioli, E. F., Mansur, A. A. P., Vasconcelos, W. L., Mansur, H. S. (2009). Preparation and characterization of chitosan/poly(vinyl alcohol) chemically crosslinked blends for biomedical applications. Carbohydrate Polymers, 76, 472-481. DOI 10.1016/j.carbpol.2008.11.015.

35. Bee, S. T., Sin, L. T., Khor, S. L., Lim, K. S., Rahmat, A. R. (2017). Enhancement of mechanical and thermal properties of (Poly[vinyl alcohol])-Dialdehyde starch composites via the incorporation of montmorillonite nanofillers. Journal of Vinyl and Additive Technology, 23, E128-E141. DOI 10.1002/vnl.21517.

36. Dou, Y., Zhang, B., He, M., Yin, G., Cui, Y. et al. (2015). Keratin/Polyvinyl alcohol blend films cross-linked by dialdehyde starch and their potential application for drug release. Polymers, 7, 580-591. DOI 10.3390/ polym7030580.

37. Teng, B., Jian, X., Gao, Y., Chen, W. (2016). Comparison of polyflavonoids in bayberry tanning effluent and commercial bayberry tannin: Prerequisite information for vegetable tanning effluent recycling. Journal of Cleaner Production, 112, 972-979. DOI 10.1016/j.jclepro.2015.09.005. 
38. Fu, Y., Qiao, L., Cao, Y., Zhou, X., Liu, Y. et al. (2014). Structural elucidation and antioxidant activities of proanthocyanidins from Chinese bayberry (Myrica rubra sieb. et zucc.) leaves. PLoS One, 9, e96162. DOI 10.1371/journal.pone.0096162.

39. Lim, M., Kwon, H., Kim, D., Seo, J., Han, H. et al. (2015). Highly-enhanced water resistant and oxygen barrier properties of cross-linked poly(vinyl alcohol) hybrid films for packaging applications. Progress in Organic Coatings, 85, 68-75. DOI 10.1016/j.porgcoat.2015.03.005.

40. Chowdhury, S., Teoh, Y. L., Ong, K. M., Rafflisman Zaidi, N. S., Mah, S. K. (2020). Poly(vinyl) alcohol crosslinked composite packaging film containing gold nanoparticles on shelf life extension of banana. Food Packaging and Shelf Life, 24, 100463. DOI 10.1016/j.fpsl.2020.100463.

41. Liao, J., Brosse, N., Pizzi, A., Hoppe, S., Zhou, X. et al. (2020). Characterization and 3D printability of poly(lactic acid)/acetylated tannin composites. Industrial Crops and Products, 149, 112320. DOI 10.1016/j. indcrop.2020.112320.

42. Floegel, A., Kim, D. O., Chung, S. J., Koo, S. I., Chun, O. K. (2011). Comparison of ABTS/DPPH assays to measure antioxidant capacity in popular antioxidant-rich US foods. Journal of Food Composition and Analysis, 24, 1043-1048. DOI 10.1016/j.jfca.2011.01.008.

43. Luo, J., Zuo, D., Deng, Z., Ji, A., Xia, G. (2020). Effects of heat treatment and Tea polyphenols on the structure and properties of polyvinyl alcohol nanofiber films for food packaging. Coatings, 10, 49. DOI 10.3390/ coatings 10010049 . 